\title{
Photometric Investigation of the Dwarf Nova OT J213806.6+261957 in Pegasus
}

\author{
P. O. Zemko ${ }^{1}$, M. V. Andreev ${ }^{2}$, D. Chochol ${ }^{3}$, N. A. Katysheva ${ }^{1}$, \\ and S. Y. Shugarov ${ }^{3,1}$ \\ ${ }^{1}$ Sternberg State Astronomical Institute, \\ 13, University Avenue, 119992, Moscow, Russia, email: polina.zemko@gmail.com \\ ${ }^{2}$ Terskol Branch of the Astronomical Institute of the Russia Academy of Sciences, \\ 48, Piatnitskaya str., 119017, Moscow, Russia \\ ${ }^{3}$ Astronomical Institute of the Slovak Academy of Sciences, \\ 05960 Tatranská Lomnica, Slovakia
}

\begin{abstract}
Our $U B V(R I)_{C}$ CCD photometry of a WZ Sge-type dwarf nova OT J213806.6+ 261957, discovered on May 6, 2010 at $8.4 \mathrm{mag}$, is presented. The analysis of our $\sim 10000 \mathrm{CCD}$ images, taken at observatories in Russia, Ukraine and Slovakia during May-November 2010, revealed the presence of ordinary and late superhumps. Fast brightness variations (flickering) on a time scale of minutes were also detected.
\end{abstract}

Keywords. stars: dwarf novae

Our $U B V R_{C}$ light curves of the DN Pegasi 2010, together with the VSNET preoutburst data, are presented in Figure 1. We removed the light of the close optical component that significantly complicated measurements. The light curve of the object after outburst $(\mathrm{AO})$ declined by $2^{m}(V)$ in 13 days and reached its quiescent stage $(V$ $\sim 16.3 \mathrm{mag})$ in about $\sim 120$ days. Nevertheless, night to night brightness variations with the amplitude $0.2-0.4 \mathrm{mag}$ remained. The types of superhumps are indicated in Figure 1. We determined the superhump periods as follows: $0^{d} .05494$ (9-14 days AO), $0^{d} .05512\left(17-21 \mathrm{~d}\right.$ AO), $0^{d} .05485$ (24-35 d AO), $0^{d} .05268$ : (111-145 d AO) and $0^{d} .05435$ (159-174 d AO). The amplitude of irregular variability on a time-scale of 10-30 minutes increased from $0.05 \mathrm{mag}$ (9-20 d AO) to 0.2-0.3 mag (24-35 d AO and 159-192 d AO). The light curves of the object during the selected nights are shown in the inner graphs.

The track of the DN Pegasi 2010 in a two-color diagram is shown in Figure 2. The object moved from its position close to the main sequence (15-23 d AO) to the position

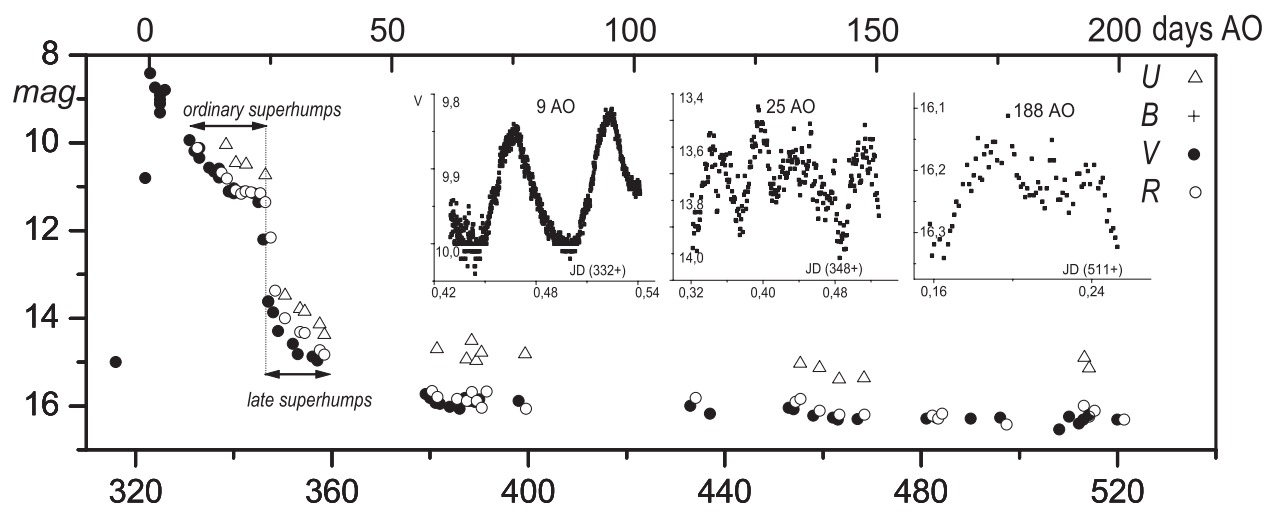

Figure 1. $U B V R_{C}$-light curves and $V$ light curves at selected nights. 


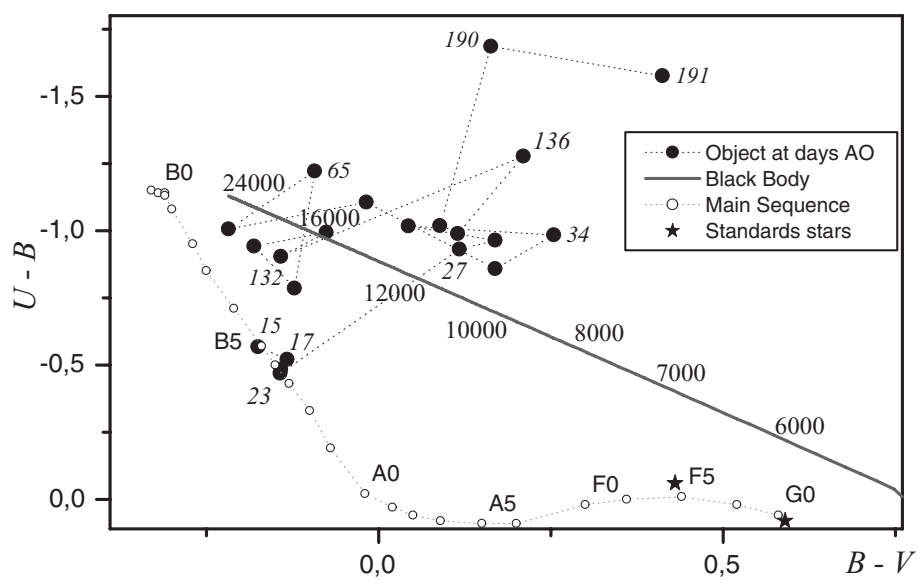

Figure 2. The track of the object in a two-color diagram.

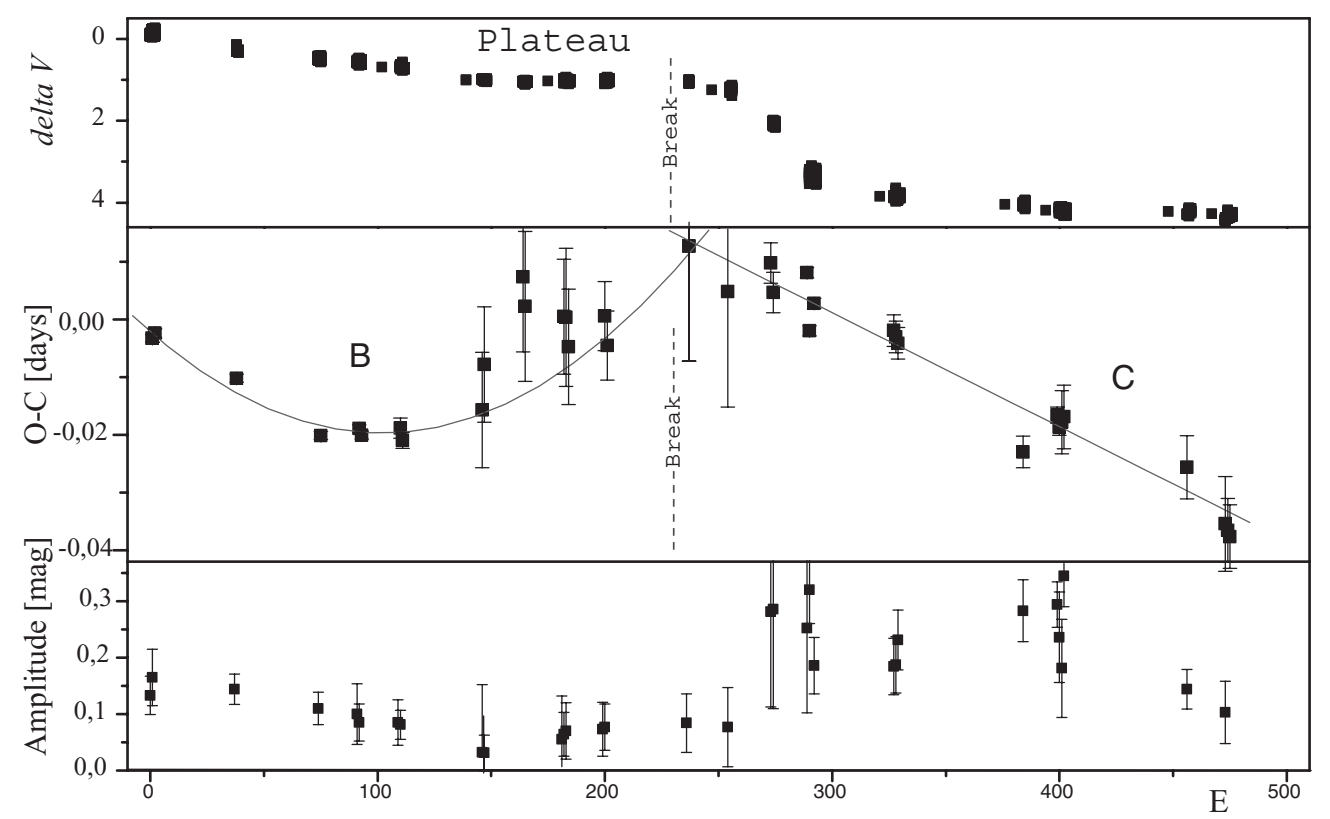

Figure 3. Schematic $\Delta V$-light curve of the DN Pegasi 2010, the O-C residuals and the amplitude of superhumps as a function of number of orbital cycles from JD 2455332.467.

around the black body sequence (27-132 d AO), when its brightness rapidly faded and a significant UV excess appeared. During the outburst the color temperature increased from 10,000 to 24,000 K. The UV excess reached -1.7 mag $~ 190$ days AO.

The amplitudes of superhumps during the stages "B" (ordinary superhumps) and "C" (late superhumps) are presented in Figure 3. For the calculation of $\mathrm{O}-\mathrm{C}$ residuals, we took the mean period $P=0^{d} .055106$ and obtained $\dot{P} / P=+6.2 \cdot 10^{-5}$. The parabolic form of the O-C curve changes to a linear one at JD 2455345 after the "period break". This behavior is typical for the most of the WZ Sge-type and SU UMa-type stars.

More detailed analysis of the object will be published in the Contributions of the Astronomical Observatory Skalnaté Pleso. 